\title{
Numerical method, existence and uniqueness for thermoelasticity system with moving boundary
}

\author{
M.A. RINCON ${ }^{1}$, B.S. SANTOS ${ }^{2}$ and J. LÍMACO ${ }^{2}$ \\ ${ }^{1}$ Instituto de Matemática, Universidade Federal do Rio de Janeiro \\ 2 Instituto de Matemática, Universidade Federal Fluminense \\ Rio de Janeiro, Brazil
}

E-mails: rincon@dcc.ufrj.br / beatrizferrel@ig.com.br / juanbrj@ hotmail.com

\begin{abstract}
In this work, we are interested in obtaining existence, uniqueness of the solution and an approximate numerical solution for the model of linear thermoelasticity with moving boundary. We apply finite element method with finite difference for evolution in time to obtain an approximate numerical solution. Some numerical experiments were presented to show the moving boundary's effects for problems in linear thermoelasticity.
\end{abstract}

Mathematical subject classification: 35A05, 35A40, 65M60, 65M06.

Key words: thermoelasticity system; moving boundary; finite element method; finite difference method.

\section{Introduction}

Let $Q_{t}=\left\{(x, t) \in \mathbb{R}^{2} ; \alpha(t)<x<\beta(t), 0<t<T\right\}$ be the non-cylindrical domain with boundary

$$
\Sigma_{t}=\bigcup_{0<t<T}\{\alpha(t), \beta(t)\} \times\{t\}
$$

\#625/04. Received: 03/III/04. Accepted: 06/I/05. 
and consider the following problem:

$$
\text { (I) }\left[\begin{array}{lr}
\frac{\partial^{2} u}{\partial t^{2}}-\frac{\partial^{2} u}{\partial x^{2}}+\eta_{1} \frac{\partial \theta}{\partial x}=0, & \forall(x, t) \in Q_{t} \\
\frac{\partial \theta}{\partial t}-k \frac{\partial^{2} \theta}{\partial x^{2}}+\eta_{2} \frac{\partial^{2} u}{\partial x \partial t}=0, & \forall(x, t) \in Q_{t}, \\
u=\theta=0, & \forall(x, t) \in \Sigma_{t}, \\
u(x, 0)=u_{0}(x), & \frac{\partial u}{\partial t}(x, 0)=u_{1}(x), \\
\theta(x, 0)=\theta_{0}(x) ; & \alpha(0)<x<\beta(0) .
\end{array}\right.
$$

Existence and uniqueness of linear and nonlinear elasticity in a bounded or an unbounded cylindrical domain, has been studied by several authors, among them, [4] and [5].

In this work, we will investigate existence, uniqueness and approximate solution of the problem (I). We will also show the influence of moving boundary employing numerical examples. For this we consider the following hypotheses:

H1: $\alpha, \beta \in C^{2}([0, T) ; \mathbb{R})$, with $0<\gamma_{0}=\min _{0 \leq t \leq T} \gamma(t)$, where $\gamma(t)=\beta(t)-\alpha(t)$,

H2: $\exists k_{1} \in \mathbb{R}$, such that, $0<k_{1}<1-\left(\alpha^{\prime}(t)+\gamma^{\prime}(t) y\right)^{2}, \quad$ for $0 \leq t \leq T$ and $0 \leq y \leq 1$.

H3: $k>0$, and $\eta_{1} \cdot \eta_{2}>0$.

We will now consider a change of variables to transform the domain $Q_{t}$ into a cylindrical domain $Q$. Observe that, when $(x, t)$ varies in $Q_{t}$ the point $(y, t)$ of $\mathbb{R}^{2}$, with $y=(x-\alpha(t)) / \gamma(t)$ varies in the cylinder $Q=(0,1) \times(0, T)$. Thus, we define the application

$$
\begin{gathered}
\mathcal{T}: Q_{t} \rightarrow Q=(0,1) \times(0, T) \\
(x, t) \mapsto(y, t)=\left(\frac{x-\alpha(t)}{\gamma(t)}, t\right) .
\end{gathered}
$$

The application $\mathcal{T}$ belongs to $C^{2}$ and its inverse $\mathcal{T}^{-1}$ is also $C^{2}$. The transformation of a moving boundary domain to a domain with fixed boundary has been employed elsewhere (see [2, 10, 11]). 
Doing the change of variable $v(y, t)=u(\alpha(t)+\gamma(t) y, t)$ and $\phi(y, t)=$ $\theta(\alpha(t)+\gamma(t) y, t)$ and applying to the problema (I), we obtain the following equivalent problem defined in a fixed cylindrical domain:

$$
\left[\begin{array}{cl}
\frac{\partial^{2} v}{\partial t^{2}}-\frac{\partial}{\partial y}\left(a_{1}(y, t) \frac{\partial v}{\partial y}\right)+a_{2}(t) \frac{\partial \phi}{\partial y} & \\
+a_{3}(y, t) \frac{\partial^{2} v}{\partial y \partial t}+a_{4}(y, t) \frac{\partial v}{\partial y}=0, & \text { in } Q \\
\frac{\partial \phi}{\partial t}-b_{1}(t) \frac{\partial^{2} \phi}{\partial y^{2}}+b_{2}(t) \frac{\partial^{2} v}{\partial y \partial t}+b_{3}(y, t) \frac{\partial \phi}{\partial y} & \\
+b_{4}(t) \frac{\partial v}{\partial y}+b_{5}(y, t) \frac{\partial^{2} v}{\partial y^{2}}=0, & \text { in } Q \\
v=\phi=0 ; & \forall(y, t) \in \Sigma, \\
v(y, 0)=v_{0}(y), \quad \frac{\partial v}{\partial t}(y, 0)=v_{1}(y), & \text { for } 0<y<1 . \\
\phi(y, 0)=\phi_{0}(y), &
\end{array}\right.
$$

where

$$
\begin{array}{lll}
b_{1}(t)=k / \gamma(t)^{2}, & b_{2}(t)=\eta_{2} / \gamma(t), & b_{3}(y, t)=-\left(\alpha^{\prime}(t)+\gamma^{\prime}(t) y\right) / \gamma(t), \\
b_{4}(t)=-\gamma^{\prime}(t) / \gamma(t)^{2}, & b_{5}(y, t)=b_{3}(y, t) / \gamma(t), & a_{1}(y, t)=1 / \gamma(t)^{2}-\left(b_{3}(y, t)\right)^{2}, \\
a_{2}(t)=\eta_{1} / \gamma(t), & a_{3}(y, t)=2 b_{3}(y, t), & a_{4}(y, t)=-\left(\alpha^{\prime \prime}(t)+\gamma^{\prime \prime}(t) y\right) / \gamma(t) .
\end{array}
$$

Let $(()),,\|\cdot\|$ and $(),,|\cdot|$, be respectively the scalar product and the norms in $H_{0}^{1}(0,1)$ and $L^{2}(0,1)$. We denote by $a_{1}(t, v, w)$ and $b_{1}(t, v, w)$ the bilinear forms, continuous, symmetric and coercive, defined in $H_{0}^{1}(0,1)$ by

$$
\begin{aligned}
& a_{1}(t, v, w)=\int_{0}^{1} a_{1}(y, t) \frac{\partial v}{\partial y} \frac{\partial w}{\partial y} d y, \\
& b_{1}(t, v, w)=\int_{0}^{1} b_{1}(t) \frac{\partial v}{\partial y} \frac{\partial w}{\partial y} d y .
\end{aligned}
$$

\section{Existence and uniqueness}

We shall first establish the existence and uniqueness of problem (II) in Theorem 2 as auxiliary and then prove the following Theorem 1 of the original problem (I). 
Theorem 1. Under the hypotheses $(\mathrm{H} 1),(\mathrm{H} 2)$ and $(\mathrm{H} 3)$ and given the initial data

$$
\left\{u_{0}, \theta_{0}\right\} \in H_{0}^{1}\left(\Omega_{0}\right) \cap H^{2}\left(\Omega_{0}\right), \quad u_{1} \in H_{0}^{1}\left(\Omega_{0}\right),
$$

there exist functions $\{u ; \theta\}: Q_{t} \rightarrow \mathbb{R}$, solution of Problem (I) in $Q_{t}$, satisfying the following conditions:

1. $u \in L^{\infty}\left(0, T ; H_{0}^{1}\left(\Omega_{t}\right) \cap H^{2}\left(\Omega_{t}\right)\right), \quad u^{\prime} \in L^{\infty}\left(0, T ; H_{0}^{1}\left(\Omega_{t}\right)\right)$, $u^{\prime \prime} \in L^{\infty}\left(0, T ; L^{2}\left(\Omega_{t}\right)\right)$,

2. $\theta \in L^{2}\left(0, T ; H_{0}^{1}\left(\Omega_{t}\right) \cap H^{2}\left(\Omega_{t}\right)\right), \quad \theta^{\prime} \in L^{2}\left(0, T ; H_{0}^{1}\left(\Omega_{t}\right)\right)$.

Theorem 2. Under the hypotheses (H1), (H2) and (H3) and given the initial data

$$
\left\{v_{0}, \phi_{0}\right\} \in H_{0}^{1}(0,1) \cap H^{2}(0,1), \quad v_{1} \in H_{0}^{1}(0,1),
$$

there exists functions $\{v ; \phi\}: Q \rightarrow \mathbb{R}$, solution of Problem (II) in $Q$, satisfying the following conditions:

1. $v \in L^{\infty}\left(0, T ; H_{0}^{1}(0,1) \cap H^{2}(0,1)\right), \quad v^{\prime} \in L^{\infty}\left(0, T ; H_{0}^{1}(0,1)\right)$, $v^{\prime \prime} \in L^{\infty}\left(0, T ; L^{2}(0,1)\right)$,

2. $\phi \in L^{2}\left(0, T ; H_{0}^{1}(0,1)\right) \cap H^{2}(0,1), \quad \phi^{\prime} \in L^{2}\left(0, T ; H_{0}^{1}(0,1)\right)$.

Proof of Theorem 2. To prove the theorem, we introduce the approximate solutions. Let $T>0$ and denote by $V_{m}$ the subspace spanned by $\left\{w_{1}, w_{2}, \ldots, w_{m}\right\}$, where $\left\{w_{\nu}, \lambda_{\nu} ; v=1, \cdots m\right\}$ are solutions of the spectral problem $\left(\left(w_{i}, v\right)\right)=$ $\mu\left(w_{i}, v\right), \forall v \in H_{0}^{1}(0,1)$. If $\left\{v_{m} ; \phi_{m}\right\} \in V_{m}$ then it can be represented by

$$
v_{m}=\sum_{\nu=1}^{m} d_{\nu m}(t) w_{\nu}(y), \quad \phi_{m}=\sum_{\nu=1}^{m} g_{\nu m}(t) w_{\nu}(y) .
$$


Let us consider $\left\{v_{m} ; \phi_{m}\right\}$ solutions of the system of ordinary differential equations,

$$
(\text { III })\left[\begin{array}{c}
\left(v_{m}^{\prime \prime}, w\right)+a_{1}\left(t, v_{m}, w\right)+a_{2}\left(\frac{\partial \phi_{m}}{\partial y}, w\right) \\
+\left(a_{3} \frac{\partial v_{m}^{\prime}}{\partial y}, w\right)+\left(a_{4} \frac{\partial v_{m}}{\partial y}, w\right)=0, \\
\left(\phi_{m}^{\prime}, w\right)+b_{1}\left(t, \phi_{m}, w\right)+b_{2}\left(\frac{\partial v_{m}^{\prime}}{\partial y}, w\right)+\left(b_{3} \frac{\partial \phi_{m}}{\partial y}, w\right) \\
+\left(2 b_{4} \frac{\partial v_{m}}{\partial y}, w\right)+\left(b_{5} \frac{\partial v_{m}}{\partial y}, \frac{\partial w}{\partial y}\right)=0, \\
v_{m}(0)=v_{0 m} \rightarrow v_{0}, \quad \text { in } \quad H_{0}^{1}(0,1) \cap H^{2}(0,1), \\
v_{m}^{\prime}(0)=v_{1 m} \rightarrow v_{1} \quad \text { in } \quad H_{0}^{1}(0,1), \\
\phi_{m}(0)=\phi_{0 m} \rightarrow \phi_{0} \quad \text { in } \quad H_{0}^{1}(0,1) \cap H^{2}(0,1),
\end{array}\right.
$$

where $w \in V_{m}$. The system (III) has local solution in the interval $\left(0, T_{m}\right)$. To extend the local solution to the interval $(0, T)$ independent of $m$, the following estimates are necessary:

\section{A priori estimate}

Taking $w=v_{m}^{\prime}$ and $w=\phi_{m}$ in the equation (III) $)_{1}$ and (III) $)_{2}$, respectively, we get

$$
\begin{gathered}
\frac{1}{2} \frac{d}{d t}\left|v_{m}^{\prime}\right|^{2}+a_{1}\left(t, v_{m}, v_{m}^{\prime}\right)+a_{2}\left(\frac{\partial \phi_{m}}{\partial y}, v_{m}^{\prime}\right) \\
+\left(a_{3} \frac{\partial v_{m}^{\prime}}{\partial y}, v_{m}^{\prime}\right)+\left(a_{4} \frac{\partial v_{m}}{\partial y}, v_{m}^{\prime}\right)=0 \\
\frac{1}{2} \frac{d}{d t}\left|\phi_{m}\right|^{2}+b_{1}\left(t, \phi_{m}, \phi_{m}\right)+b_{2}\left(\frac{\partial v_{m}^{\prime}}{\partial y}, \phi_{m}\right)+\left(b_{3} \frac{\partial \phi_{m}}{\partial y}, \phi_{m}\right) \\
+2 b_{4}\left(\frac{\partial v_{m}}{\partial y}, \phi_{m}\right)+\left(b_{5} \frac{\partial v_{m}}{\partial y}, \frac{\partial \phi_{m}}{\partial y}\right)=0 .
\end{gathered}
$$


Note that, we have the following relations:

$$
\begin{aligned}
& a_{1}\left(t, v_{m}, v_{m}^{\prime}\right)=\frac{1}{2} \frac{d}{d t} a_{1}\left(t, v_{m}, v_{m}\right)-\frac{1}{2}\left(a_{1}^{\prime} \frac{\partial v_{m}}{\partial y}, \frac{\partial w}{\partial y}\right), \\
& a_{2}\left(\frac{\partial \phi_{m}}{\partial y}, v_{m}^{\prime}\right)=-\frac{\eta_{1}}{\eta_{2}} b_{2}\left(\frac{\partial v_{m}^{\prime}}{\partial y} \phi_{m}\right), \\
& \left(a_{3} \frac{\partial v_{m}^{\prime}}{\partial y}, v_{m}^{\prime}\right)=-\frac{\gamma^{\prime}}{\gamma}\left|v_{m}^{\prime}\right|^{2}, \\
& \left(b_{3} \frac{\partial \phi_{m}}{\partial y}, \phi_{m}\right)=\frac{\gamma^{\prime}}{2 \gamma}\left|\phi_{m}\right|^{2}, \\
& \left|\left(b_{5} \frac{\partial v_{m}}{\partial y}, \frac{\partial \phi_{m}}{\partial y}\right)\right| \leq c\left\|v_{m}\right\|^{2}+\frac{1}{2} b_{1}\left(t, \phi_{m}, \phi_{m}\right) .
\end{aligned}
$$

Multiplying (4) by $\left(\eta_{2} / \eta_{1}\right)$, adding it to (5) and using (6) we have

$$
\begin{aligned}
& \frac{\eta_{2}}{2 \eta_{1}} \frac{d}{d t}\left(\left|v_{m}^{\prime}\right|^{2}+a_{1}\left(t, v_{m}, v_{m}\right)+\frac{\eta_{1}}{\eta_{2}}\left|\phi_{m}\right|^{2}\right)+b_{1}\left(t, \phi_{m}, \phi_{m}\right) \\
& \leq C\left(\left|v_{m}^{\prime}\right|^{2}+\left\|v_{0 m}\right\|^{2}+\left|\phi_{m}\right|^{2}\right) .
\end{aligned}
$$

Knowing that $a_{1}(t, v, w)$ and $b_{1}(t, v, w)$ are coercive forms, by integrating (7) and applying the Gronwall's inequality, we get

$$
\left|v_{m}^{\prime}\right|^{2}+\left\|v_{m}\right\|^{2}+\left|\phi_{m}\right|^{2}+\int_{0}^{t}\left\|\phi_{m}\right\|^{2} \leq c_{1}\left(\left|v_{1 m}\right|^{2}+\left\|v_{0 m}\right\|^{2}+\left|\phi_{0 m}\right|^{2}\right) e^{c_{2} T}
$$

\section{Second estimate}

Taking the derivative with respect to $t$, of approximate system (III) $)_{1,2}$, and also $w=v_{m}^{\prime \prime}, w=\phi_{m}^{\prime}$, respectively, we obtain

$$
\begin{aligned}
\left(v_{m}^{\prime \prime \prime}, v_{m}^{\prime \prime}\right) & +a_{1}\left(t, v_{m}^{\prime}, v_{m}^{\prime \prime}\right)+a_{2}\left(\frac{\partial \phi_{m}^{\prime}}{\partial y}, v_{m}^{\prime \prime}\right)+\left(a_{3} \frac{\partial v_{m}^{\prime \prime}}{\partial y}, v_{m}^{\prime \prime}\right) \\
& +\left(\left(a_{3}^{\prime}+a_{4}\right) \frac{\partial v_{m}^{\prime}}{\partial y}, v_{m}^{\prime \prime}\right)+a_{1}^{\prime}\left(t, v_{m}, v_{m}^{\prime \prime}\right)+\left(a_{2}^{\prime} \frac{\partial \phi_{m}}{\partial y}, v_{m}^{\prime \prime}\right) \\
& +\left(a_{4}^{\prime} \frac{\partial v_{m}}{\partial y}, v_{m}^{\prime \prime}\right)=0
\end{aligned}
$$


and

$$
\begin{aligned}
d s\left(\phi_{m}^{\prime \prime}, \phi_{m}^{\prime}\right) & +b_{1}\left(t, \phi_{m}^{\prime}, \phi_{m}^{\prime}\right)+b_{2}\left(\frac{\partial v_{m}^{\prime \prime}}{\partial y}, \phi_{m}^{\prime}\right)+\left(b_{3} \frac{\partial \phi_{m}^{\prime}}{\partial y}, \phi_{m}^{\prime}\right) \\
& +\left(2 b_{4}+b_{2}^{\prime}\right)\left(\frac{\partial v_{m}^{\prime}}{\partial y}, \phi_{m}^{\prime}\right)-\left(b_{5} \frac{\partial v_{m}^{\prime}}{\partial y}, \frac{\partial \phi_{m}^{\prime}}{\partial y}\right) \\
& +b_{1}^{\prime}\left(t, \phi_{m}, \phi_{m}^{\prime}\right)+\left(b_{3}^{\prime} \frac{\partial \phi_{m}}{\partial y}, \phi_{m}^{\prime}\right)+2 b_{4}^{\prime}\left(\frac{\partial v_{m}}{\partial y}, \phi_{m}^{\prime}\right) \\
& +\left(b_{5}^{\prime} \frac{\partial v_{m}}{\partial y}, \frac{\partial \phi_{m}^{\prime}}{\partial y}\right)=0 .
\end{aligned}
$$

We also have the following relations:

$$
\begin{aligned}
& a_{1}\left(t, v_{m}^{\prime}, v_{m}^{\prime \prime}\right)=\frac{1}{2} \frac{d}{d t} a_{1}^{\prime}\left(t, v_{m}^{\prime}, v_{m}^{\prime}\right)-\frac{1}{2} a_{1}^{\prime}\left(t, v_{m}^{\prime}, v_{m}^{\prime}\right), \\
& \left(a_{3} \frac{\partial v_{m}^{\prime \prime}}{\partial y}, v_{m}^{\prime \prime}\right)=\frac{\gamma^{\prime}}{\gamma}\left|v_{m}^{\prime \prime}\right|^{2}, \\
& a_{1}^{\prime}\left(t, v_{m}, v_{m}^{\prime \prime}\right)=\frac{d}{d t}\left(a_{1}^{\prime} \frac{\partial v_{m}}{\partial y}, \frac{\partial v_{m}^{\prime}}{\partial y}\right)-\left(a_{1}^{\prime \prime} \frac{\partial v_{m}}{\partial y}, \frac{\partial v_{m}^{\prime}}{\partial y}\right)-\left(a_{1}^{\prime} \frac{\partial v_{m}^{\prime}}{\partial y}, \frac{\partial v_{m}^{\prime}}{\partial y}\right), \\
& a_{2}\left(\frac{\partial \phi_{m}^{\prime}}{\partial y}, v_{m}^{\prime \prime}\right)=-\frac{\eta_{1} \cdot b_{2}}{\eta_{2}}\left(\frac{\partial v_{m}^{\prime \prime}}{\partial y}, \phi_{m}^{\prime}\right), \\
& \left(b_{3} \frac{\partial \phi_{m}^{\prime}}{\partial y}, \phi_{m}^{\prime}\right)=\frac{1}{2} \frac{\gamma^{\prime}}{\gamma}\left|\phi_{m}^{\prime}\right|^{2} \\
& \left|\left(a_{1}^{\prime} \frac{\partial v_{m}}{\partial y}, \frac{\partial v_{m}^{\prime}}{\partial y}\right)\right| \leq C\left\|v_{m}\right\|^{2}+\frac{\eta_{2}}{4 \eta_{1}} a_{1}\left(t, v_{m}^{\prime}, v_{m}^{\prime}\right) .
\end{aligned}
$$

Multiplying (9) by $\left(\eta_{1} / \eta_{2}\right)$, adding it to (10) and using (11), we obtain

$$
\begin{aligned}
& \frac{\eta_{2}}{2 \eta_{1}} \frac{d}{d t}\left\{\left|v_{m}^{\prime \prime}\right|^{2}+a_{1}\left(t, v_{m}^{\prime}, v_{m}^{\prime}\right)+\left(a_{1}^{\prime} \frac{\partial v_{m}}{\partial y}, \frac{\partial v_{m}^{\prime}}{\partial y}\right)+\frac{\alpha}{\beta}\left|\phi_{m}^{\prime}\right|\right\} \\
& \quad+b_{1}\left(t, \phi_{m}^{\prime}, \phi_{m}^{\prime}\right) \leq C\left(\left\|v_{m}\right\|^{2}+\left\|v_{m}^{\prime}\right\|^{2}+\left|v_{m}^{\prime \prime}\right|^{2}+\left\|\phi_{m}\right\|^{2}+\left|\phi_{m}^{\prime}\right|^{2}\right) .
\end{aligned}
$$

From (III) $)_{1,5},\left|v_{m}^{\prime \prime}(0)\right|^{2}$ and $\left|\phi_{m}^{\prime}(0)\right|^{2}$ are bounded. Hence, by integrating (12) with respect $t$ and applying the Gronwall's inequality, we get

$$
\left\|v_{m}^{\prime}\right\|^{2}+\left|v_{m}^{\prime \prime}\right|^{2}+\left|\phi_{m}^{\prime}\right|^{2}+\int_{0}^{t}\left\|\phi_{m}^{\prime}\right\|^{2} \leq C .
$$




\section{Third estimate}

Taking $w=-\partial^{2} v_{m} / \partial y^{2}$ and $w=-\partial^{2} \phi_{m} / \partial y^{2}$, in the approximate system (III) $)_{1,2}$, we have

$$
\begin{aligned}
\left(v_{m}^{\prime \prime},-\frac{\partial^{2} v_{m}}{\partial y^{2}}\right) & +a_{1}\left(t, v_{m},-\frac{\partial^{2} v_{m}}{\partial y^{2}}\right)+a_{2}\left(\frac{\partial \phi_{m}}{\partial y},-\frac{\partial^{2} v_{m}}{\partial y^{2}}\right) \\
& +\left(a_{3} \frac{\partial v_{m}^{\prime}}{\partial y},-\frac{\partial^{2} v_{m}}{\partial y^{2}}\right)+\left(a_{4} \frac{\partial v_{m}}{\partial y},-\frac{\partial^{2} v_{m}}{\partial y^{2}}\right)=0
\end{aligned}
$$

and

$$
\begin{aligned}
\left(\phi_{m}^{\prime},-\frac{\partial^{2} \phi_{m}}{\partial y^{2}}\right) & +b_{1}\left(t, \phi_{m},-\frac{\partial^{2} \phi_{m}}{\partial y^{2}}\right)+b_{2}\left(\frac{\partial v_{m}^{\prime}}{\partial y},-\frac{\partial^{2} \phi_{m}}{\partial y^{2}}\right) \\
& +2 b_{4}\left(\frac{\partial v_{m}}{\partial y},-\frac{\partial^{2} \phi_{m}}{\partial y^{2}}\right)+\left(b_{5} \frac{\partial v_{m}}{\partial y},-\frac{\partial^{3} \phi_{m}}{\partial y^{3}}\right)=0 .
\end{aligned}
$$

Note that, we have the following equalities:

$$
\begin{aligned}
& a_{1}\left(t, v_{m},-\frac{\partial^{2} v_{m}}{\partial y^{2}}\right)=a_{1}\left(t, \frac{\partial v_{m}}{\partial y}, \frac{\partial v_{m}}{\partial y}\right)+\left(\frac{\partial a_{1}}{\partial y} \frac{\partial v_{m}}{\partial y}, \frac{\partial^{2} v_{m}}{\partial y^{2}}\right) \\
& b_{1}\left(t, \phi_{m},-\frac{\partial^{2} \phi_{m}}{\partial y^{2}}\right)=b_{1}\left(t, \frac{\partial \phi_{m}}{\partial y}, \frac{\partial \phi_{m}}{\partial y}\right) \\
& \left(b_{5} \frac{\partial v_{m}}{\partial y},-\frac{\partial^{3} \phi_{m}}{\partial y^{3}}\right)=\left(b_{5} \frac{\partial^{2} v_{m}}{\partial y^{2}}, \frac{\partial^{2} \phi_{m}}{\partial y^{2}}\right)-\left(\frac{\partial b_{5}}{\partial y} \frac{\partial v_{m}}{\partial y}, \frac{\partial^{2} \phi_{m}}{\partial y^{2}}\right) .
\end{aligned}
$$

From (14), (15) and (16) and since $a_{1}(t, v, w)$ and $b_{1}(t, v, w)$ are coercive forms, we obtain

$$
\begin{aligned}
& \left|\frac{\partial^{2} v_{m}}{\partial y^{2}}\right|^{2} \leq c_{6}\left(\left\|\phi_{m}\right\|^{2}+\left|v_{m}^{\prime \prime}\right|^{2}+\left\|v_{m}^{\prime}\right\|^{2}+\left\|v_{m}\right\|^{2}\right), \\
& \left|\frac{\partial^{2} \phi_{m}}{\partial y^{2}}\right|^{2} \leq c_{7}\left(\left|\phi_{m}^{\prime}\right|^{2}+\left\|\phi_{m}\right\|^{2}+\left|v_{m}^{\prime \prime}\right|^{2}+\left\|v_{m}^{\prime}\right\|^{2}+\left\|v_{m}\right\|^{2}\right) .
\end{aligned}
$$

The estimates obtained in (8), (13), (17) and (18), permit us to pass the limits in the approximate system (III) $)_{1,2}$ in the Galerkin method and hence, we have proved the existence of solutions $\{v, \phi\}$ in the sense defined in Theorem 2 . 


\section{Uniqueness of solution}

Let $\{\hat{v}, \hat{\phi}\}$ and $\{\widetilde{v}, \widetilde{\phi}\}$ be two solutions of Problem (II). Then $v=\hat{v}-\widetilde{v}$ and $\phi=\hat{\phi}-\widetilde{\phi}$ are also solutions of Problem (II), with null initial conditions. Then, multiplying the equation (II) $)_{1,2}$, respectively by $\left(\eta_{1} / \eta_{2}\right) v$ and $\phi$, we obtain

$$
\left|v^{\prime}\right|^{2}+\|v\|^{2}+|\phi|^{2} \leq c \int_{0}^{t}\left(\left|v^{\prime}\right|^{2}+\|v\|^{2}+|\phi|^{2}\right) .
$$

From Gronwall Lemma, we have $\left|v^{\prime}\right|^{2}+\|v\|^{2}+|\phi|^{2}=0$ and therefore, we conclude that $v=\phi=0$ for all $0<t<T$. This completes the proof of Theorem 2.

\section{The original problem (I)}

Now let us restate the previous results for the original problem (I) in order to prove Theorem 1.

Proof of Theorem 1. Let $\{v, \phi\}$ be a solution of Problem (II), with initial data given by

$$
\begin{aligned}
& v_{0}(y)=u_{0}(\alpha(0)+\gamma(0) y), \quad \phi_{0}(y)=\theta_{0}(\alpha(0)+\gamma(0) y), \\
& v_{1}(y)=u_{1}(\alpha(0)+\gamma(0) y)+\left(\alpha^{\prime}(0)+\gamma^{\prime}(0) y\right) u_{0}^{\prime}(\alpha(0)+\gamma(0) y) .
\end{aligned}
$$

Consider the functions $u(x, t)=v(y, t)$ and $\theta(x, t)=\phi(y, t)$, where $x=$ $\alpha(t)+\gamma(t) y$. To verify that $u(x, t)$ and $\theta(x, t)$, under the hypotheses of Theorem 1 , are a solution of problem (I), it is sufficient to observe that the mapping: $(x, t) \rightarrow((x-\alpha) / \gamma, t)$ of the domain $Q_{t}$ into $Q=(0,1) \times(0, T)$ is of class $C^{2}$. Since that

$$
\begin{aligned}
& \text { 1. } \frac{\partial u^{2}}{\partial x^{2}}=\frac{1}{\gamma} \frac{\partial v^{2}}{\partial y^{2}}, \quad \eta_{1} \frac{\partial \theta}{\partial x}=a_{2} \frac{\partial \theta}{\partial y}, \\
& \text { 2. } u^{\prime \prime}=v^{\prime \prime}-\frac{\partial}{\partial y}\left(a_{1} \frac{\partial v}{\partial y}\right)+a_{3} \frac{\partial^{2} v}{\partial y \partial t}+a_{4} \frac{\partial v}{\partial y}+\frac{1}{\gamma^{2}} \frac{\partial^{2} v}{\partial y^{2}} \\
& \text { 3. } k \frac{\partial^{2} \theta}{\partial x^{2}}=b_{1} \frac{\partial^{2} \phi}{\partial y^{2}}, \quad \frac{\partial \theta}{\partial t}=\frac{\partial \phi}{\partial t}+b_{3} \frac{\partial \phi}{\partial y}, \\
& \text { 4. } \quad \eta_{2} \frac{\partial^{2} u}{\partial x \partial t}=b_{2} \frac{\partial^{2} v}{\partial y \partial t}+b_{4} \frac{\partial v}{\partial y}
\end{aligned}
$$


and from problem (II) we also have that $\{u, \theta\}$ satisfies the problem (I).

The regularity of $\{v(y, t), \phi(y, t)\}$ given by Theorem 2 implies that $\{u(x, t)$, $\theta(x, t)\}$ is a solution of problem (I) and the uniqueness of the solution of problem (I) is a direct consequence of the uniqueness of problem (II).

\section{Approximate solution}

Our goal in this section is the numerical implementation of approximate solutions. To obtain the numerical approximate solutions we will use both finite element method and finite difference method. Moreover, some numerical experiments will be presented to analyze the effect of the moving boundary in the thermoelasticity system.

For convenience, our numerical analysis using finite element method approximation will be based on the equivalent problem (II) in the rectangular domain, instead of the problem (I), for which the domain depends on time. We will consider, in numerical simulations, the case in which the following change in the boundary functions, $\alpha(t)=-K(t)$ and $\beta(t)=K(t)$, is assumed.

Note that, now we have

$$
Q_{t}=\left\{(x, t) \in \mathbb{R}^{2} ; x=K(t) y, y \in(-1,1), t \in(0, T)\right\}
$$

being the non-cylindrical domain with boundary

$$
\Sigma_{t}=\bigcup_{0<t<T}\{-K(t), K(t)\} \times\{t\}
$$

and consequently we have the fixed cylindrical domain $Q=(-1,1) \times(0, T)$. In this way we obtain the following relation between the functions:

$$
\begin{aligned}
& u(x, t)=v(y, t)=v\left(\frac{x}{K(t)}, t\right) \quad \text { and } \\
& \theta(x, t)=\phi(y, t)=\phi\left(\frac{x}{K(t)}, t\right) .
\end{aligned}
$$




\subsection{Variational form of the problem}

Let us consider the following variational form, given by $(\text { III })_{1,2}$,

$$
\begin{gathered}
\left(v_{m}^{\prime \prime}, w\right)+a_{1}\left(t, v_{m}, w\right)+a_{2}\left(\frac{\partial \phi_{m}}{\partial y}, w\right)+\left(a_{3} \frac{\partial v_{m}^{\prime}}{\partial y}, w\right) \\
+\left(a_{4} \frac{\partial v_{m}}{\partial y}, w\right)=0
\end{gathered}
$$

and

$$
\begin{aligned}
\left(\phi_{m}^{\prime}, w\right) & +b_{1}\left(t, \phi_{m}, w\right)+b_{2}\left(\frac{\partial v_{m}^{\prime}}{\partial y}, w\right)+\left(b_{3} \frac{\partial \phi_{m}}{\partial y}, w\right) \\
& +2 b_{4}\left(\frac{\partial v_{m}}{\partial y}, w\right)-\left(b_{5} \frac{\partial v_{m}}{\partial y}, \frac{\partial w}{\partial y}\right)=0, \quad \forall w \in V_{m}
\end{aligned}
$$

where now, using (20), the functions $b_{i}$ and $a_{i}$ are given by

$$
\begin{array}{lll}
b_{1}=k / K^{2}(t), & b_{2}=\eta_{2} / K(t), & b_{3}=-K^{\prime}(t) y / K(t), \\
b_{4}=-K^{\prime}(t) / K^{2}(t), & b_{5}=-b_{3} / K(t), & a_{1}=1 / K^{2}(t)-b_{3}^{2}, \\
a_{2}=\eta_{1} / K(t), & a_{3}=2 b_{3}, & a_{4}=-K^{\prime \prime}(t) y / K(t) .
\end{array}
$$

\section{Galerkin method and approximation}

Consider the functions $\left\{v_{m} ; \phi_{m}\right\} \in V_{m}$ defined in (3). Taking $w=\varphi_{j}(y)$ and substituting in (22) and (23), we obtain the system of ordinary equations, given by

$$
\left\{\begin{array}{l}
A d^{\prime \prime}(t)+(B(t)+E(t)) d(t)+\left(a_{2} C\right) g(t)+D(t) d^{\prime}(t)=0, \\
A g^{\prime}(t)+\left(b_{1} F+G(t)\right) g(t)+\left(b_{2} C\right) d^{\prime}(t)+\left(2 b_{4} C+R(t)\right) d(t)=0,
\end{array}\right.
$$


where

$$
\begin{array}{ll}
A=\int_{-1}^{1} \varphi_{i}(y) \varphi_{j}(y) d y, & B(t)=\int_{-1}^{1} a_{1} \frac{\partial \varphi_{i}(y)}{\partial y} \frac{\partial \varphi_{j}(y)}{\partial y} d y, \\
C=\int_{-1}^{1} \frac{\partial \varphi_{i}(y)}{\partial y} \varphi_{j}(y) d y, & D(t)=\int_{-1}^{1} a_{3} \frac{\partial \varphi_{i}(y)}{\partial y} \varphi_{j}(y) d y, \\
E(t)=\int_{-1}^{1} a_{4} \frac{\partial \varphi_{i}(y)}{\partial y} \varphi_{j}(y) d y, & F=\int_{-1}^{1} \frac{\partial \varphi_{i}(y)}{\partial y} \frac{\partial \varphi_{j}(y)}{\partial y} d y, \\
G(t)=\int_{-1}^{1} b_{3} \frac{\partial \varphi_{i}(y)}{\partial y} \varphi_{j}(y) d y, & R(t)=\int_{-1}^{1}-b_{5} \frac{\partial \varphi_{i}(y)}{\partial y} \frac{\partial \varphi_{j}(y)}{\partial y} d y .
\end{array}
$$

In (25) we have introduced

$$
d(t)=\left(d_{1}(t), \cdots d_{m}(t)\right)^{t} \quad \text { and } \quad g(t)=\left(g_{1}(t), \cdots g_{m}(t)\right)^{t}
$$

For numerical reasons, we can rewrite matrix $B(t)$ in the form $B=B^{1}+B^{2}$ by using (24), where

$$
\begin{aligned}
& B^{1}(t)=\frac{1}{K(t)^{2}} \int_{-1}^{1} \frac{\partial \varphi_{i}(y)}{\partial y} \frac{\partial \varphi_{j}(y)}{\partial y} d y \\
& B^{2}(t)=-\left(\frac{K^{\prime}(t)}{K(t)}\right)^{2} \int_{-1}^{1}(y)^{2} \frac{\partial \varphi_{i}(y)}{\partial y} \frac{\partial \varphi_{j}(y)}{\partial y} d y
\end{aligned}
$$

\subsection{Finite element approximation}

We now present a semi-discrete formulation for problem (25) using the Galerkin finite element method to discretize the spatial variable. We first applied the method to find the approximate solution of the exact solution $v(y, t)$ of the Problem (II) and later, using the transformation (21) we can obtain the approximate solution of $u(x, t)$ for the Problem (I) in the domain $Q_{t}$.

First, we divide the domain $\Omega=(0,1)$ in local domain $\Omega_{i}=\left(y_{i}, y_{i+1}\right)$. Then, $\Omega=\operatorname{int}\left(\cup_{i=1}^{m} \bar{\Omega}_{i}\right)$ and $\Omega_{i} \cap \Omega_{j}=\emptyset$, if $i \neq j$. In finite element method, the $\varphi_{i}$ are piecewise polynomials of some degree in $\Omega$ and vanish on $\partial \Omega$. More 
specifically, in this work, we have used the basis function

$$
\varphi_{i}(y)= \begin{cases}\frac{y-y_{i-1}}{h}, & \forall y \in\left[y_{i-1}, y_{i}\right] \\ \frac{y_{i+1}-y}{h}, & \forall y \in\left[y_{i}, y_{i+1}\right] \\ 0, & \forall y \notin\left[y_{i-1}, y_{i+1}\right]\end{cases}
$$

where we are considering the uniform mesh, $h=h_{i}=y_{i+1}-y_{i}, i=1,2, \ldots, m$ in the discretization in $m$-parts, with $-1=y_{1}<y_{2}<\cdots<y_{m+1}=1$. Note that, if $|i-j|>2$, then $\left(\varphi_{i}, \varphi_{j}\right)=0$, and $\left(\partial \varphi_{i} / \partial y, \partial \varphi_{j} / \partial y\right)=0$. Hence all the matrices of system are tridiagonal.

\section{Matrix calculation}

For each $\Omega_{i}$, we have to calculate each integral defined in (26), using the functions (24), (27) and its derivatives. Doing the calculus, we obtain, respectively the following elements, for each tridiagonal matrix $A, B^{1}, B^{2}, C, D, E, F, G$ and $R$ :

$$
\begin{aligned}
& a_{i i}=\frac{4}{3 m}, \quad a_{i, i+1}=a_{i+1, i}=\frac{1}{3 m}, \\
& b_{i i}^{1}=\frac{m}{K^{2}}, \quad b_{i, i+1}^{1}=b_{i+1, i}^{1}=-\frac{m}{2 K^{2}}, \\
& b_{i i}^{2}=-\frac{m\left(K^{\prime}\right)^{2}}{3 K^{2}}\left(3 y_{i}^{2}+\frac{4}{m^{2}}\right), \\
& b_{i, i+1}^{2}=b_{i+1, i}^{2}=\frac{m\left(K^{\prime}\right)^{2}}{6 K^{2}}\left(3 y_{i}^{2}+\frac{6 y_{i}}{m}+\frac{4}{m^{2}}\right) \\
& c_{i i}=0, \quad c_{i, i+1}=-\frac{1}{2}, \quad c_{i+1, i}=\frac{1}{2} \\
& d_{i i}=\frac{4 K^{\prime}}{3 m K}, \quad d_{i, i+1}=\frac{K^{\prime}}{3 K}\left(\frac{4}{m}+3 y_{i}\right), \quad d_{i+1, i}=-\frac{K^{\prime}}{3 K}\left(\frac{2}{m}+3 y_{i}\right), \\
& e_{i i}=\frac{2 K^{\prime \prime}}{3 m K}, \quad e_{i, i+1}=\frac{K^{\prime \prime}}{6 K}\left(\frac{4}{m}+3 y_{i}\right), \quad e_{i+1, i}=-\frac{K^{\prime \prime}}{6 K}\left(\frac{2}{m}+3 y_{i}\right), \\
& f_{i i}=m, \quad f_{i, i+1}=f_{i+1, i}=-\frac{m}{2} \\
& g_{i i}=\frac{2 K^{\prime}}{3 m K}, \quad g_{i, i+1}=\frac{K^{\prime}}{6 K}\left(\frac{4}{m}+3 y_{i}\right), \quad g_{i+1, i}=-\frac{K^{\prime}}{6 K}\left(\frac{2}{m}+3 y_{i}\right), \\
& r_{i, i}=-\frac{m K^{\prime} y_{i}}{K^{2}}, \quad r_{i, i+1}=r_{i+1, i}=\frac{K^{\prime}}{2 K^{2}}\left(1+m y_{i}\right),
\end{aligned}
$$




\subsection{Finite difference method}

The equation (25) represent a system of ordinary differential equations of second order and due to matrices characteristics (dependent on the variables $y$ and $t$ ) of system, obtaining the solution is not always possible. So, we will apply a numerical method to obtain the approximated solution for the system (25), using the approximate Newmark's Method (see, for instance, Hugles [7], pp 493).

Let $d^{n}=d\left(t_{n}\right)$ and $g^{n}=g\left(t_{n}\right)$ be the approximate solution of the exact solution $d(t)$ and $g(t)$ of $(25)_{1,2}$, respectively, where we denote the discrete times in the interval $[0, T]$ by $t_{n}=n \Delta t, n=0,1 \cdots N$.

For $\delta \geq 1 / 4$, with $\delta \in \mathbb{R}$, consider the following approximation

$$
\begin{aligned}
& d^{* n}=\delta d^{n+1}+(1-2 \delta) d^{n}+\delta d^{n-1} \\
& g^{* n}=\delta g^{n+1}+(1-2 \delta) g^{n}+\delta g^{n-1},
\end{aligned}
$$

and for the first and second derivative, we take the difference operator in the following form

$$
\begin{aligned}
\tau d^{n} & =\frac{d^{n+1}-d^{n-1}}{2 \Delta t}, \\
\tau g^{n} & =\frac{g^{n+1}-g^{n-1}}{2 \Delta t}, \\
\delta^{2} d^{n} & =\frac{d^{n+1}-2 d^{n}+d^{n-1}}{\Delta t^{2}}
\end{aligned}
$$

which, for this approximation the discrete error can be showed to be of order $\mathcal{O}\left(\Delta t^{2}\right)$.

\section{Coupled system}

For the system (25) $)_{1,2}$ at the discrete mesh points $t_{n}=n \Delta t$, using (29) and (30), we obtain the following coupled system:

$$
\left\{\begin{array}{l}
\widehat{A}^{n} d^{n+1}+\widehat{B}^{n} g^{n+1}=\widehat{C} d^{n}-\widehat{D} d^{n-1}-\widehat{E} g^{n}-\widehat{F} g^{n-1} \\
\widetilde{A}^{n} d^{n+1}+\widetilde{B}^{n} g^{n+1}=-\widetilde{C} d^{n}+\widetilde{D} d^{n-1}-\widetilde{E} g^{n}+\widetilde{F} g^{n-1}
\end{array}\right.
$$


where,

$$
\begin{aligned}
& \widehat{A}^{n}=A+\delta \Delta t^{2}\left(B^{1}\right)^{n}+\frac{\Delta t}{2} D^{n}, \quad \widehat{B}^{n}=a_{2}^{n} \delta \Delta t^{2} C \\
& \widehat{C}^{n}=2 A-\Delta t^{2}\left((1-2 \delta)\left(B^{1}\right)^{n}+\left(B^{2}\right)^{n}+E^{n}\right) \\
& \widehat{D}^{n}=A+\delta \Delta t^{2}\left(B^{1}\right)^{n}-\frac{\Delta t}{2} D^{n}, \quad \widehat{E}^{n}=a_{2}^{n}(1-2 \delta) \Delta t^{2} C \\
& \widehat{F}^{n}=a_{2}^{n} \delta \Delta t^{2} C, \quad \widetilde{A}_{2}^{n}{ }^{n}, \quad \widetilde{B}^{n}=\frac{A}{2}+b_{1}^{n} \delta \Delta t F \\
& \widetilde{C}^{n}=\Delta t\left(2 b_{4}^{n} C+R^{n}\right), \quad \frac{b_{2}^{n}}{2} C \\
& \widetilde{E}^{n}=\Delta t\left(b_{1}^{n}(1-2 \delta) F+G^{n}\right), \quad \widetilde{F}^{n}=\frac{A}{2}-b_{1}^{n} \delta \Delta t F
\end{aligned}
$$

To determine the solution $\left\{d^{n}, g^{n}\right\}$, the coupled system of algebraic equations (31) may be solved by iteration, as follows: To start the iteration, we first take $n=0$ in (31) and rewrite the system as

$$
\left\{\begin{array}{l}
\widehat{A}^{0} d^{1}+\widehat{B}^{0} g^{1}=\widehat{C}^{0} d^{0}-\widehat{D}^{0} d^{-1}-\widehat{E}^{0} g^{0}-\widehat{F}^{0} g^{-1} \\
\widetilde{A}^{0} d^{1}+\widetilde{B}^{0} g^{1}=-\widetilde{C}^{0} d^{0}-\widetilde{D}^{0} d^{-1}-\widetilde{E}^{0} g^{0}+\widetilde{F}^{0} g^{-1}
\end{array}\right.
$$

where the right-hand side is determined by the (starting) values, since the exact solutions $\{v(y, t) ; \phi(y, t)\}$ are known at time $t=0$ and $\left\{v^{0} ; \phi^{0}\right\}$ are just the initial values, i.e, $d^{0}=v^{0}()=.v(., 0), \quad g^{0}=\phi^{0}()=.\phi(., 0)$, where we have used (3) and (27).

We can calculate an approximation for $\left\{d^{-1} ; g^{-1}\right\}$ by the second order Taylor extrapolation of $\{v(., t) ; \phi(., t)\}$ from $t^{0}=0, v i z$,

$$
d^{-1}=d^{0}-\Delta t d^{\prime}(0)+\frac{\Delta t^{2}}{2} d^{\prime \prime}(0), \quad g^{-1}=g^{0}-\Delta t g^{\prime}(0)
$$

in which the values of $\left.d^{\prime}(0), d^{\prime \prime} 0\right)$ and $g^{\prime}(0)$ are given by

$$
d^{\prime}(0)=\frac{\partial v}{\partial t}\left(y_{i}, 0\right)=v_{1}\left(y_{i}\right), \quad d^{\prime \prime}(0)=\frac{\partial^{2} v}{\partial t^{2}}\left(y_{i}, 0\right), \quad g^{\prime}(0)=\frac{\partial \phi}{\partial t}\left(y_{i}, 0\right)
$$


calculated from the equation (II) $)_{1}$ and $(\mathbf{I I})_{2}$, at $t^{0}=0$ and the initial values $v^{0}()=.v(., 0)$ and $\phi^{0}()=.\phi(., 0)$.

The system may be solved uniquely for $\left\{d^{1}, g^{1}\right\}$, since its coefficient matrix is non singular. Having determined the values $\left\{d^{1}, g^{1}\right\}$, then for $n=1,2, \cdots N$, we obtain the approximate solution $\left\{d^{n+1}, g^{n+1}\right\}$ for the coupled system of algebraic equations (31), which can be rewritten in the form of block matrix,

$$
\left[\begin{array}{cc}
\widehat{A}^{n} & \widehat{B}^{n} \\
\widetilde{A}^{n} & \widetilde{B}^{n}
\end{array}\right]\left[\begin{array}{l}
d^{n+1} \\
g^{n+1}
\end{array}\right]=\left[\begin{array}{c}
S^{n} \\
T^{n}
\end{array}\right]
$$

where,

$$
\begin{aligned}
S^{n} & =\widehat{C} d^{n}-\widehat{D} d^{n-1}-\widehat{E} g^{n}-\widehat{F} g^{n-1} \text { and } \\
T^{n} & =-\widetilde{C} d^{n}+\widetilde{D} d^{n-1}-\widetilde{E} g^{n}+\widetilde{F} g^{n-1} .
\end{aligned}
$$

The system (34) may be solved uniquely, since the matrix is non-singular. In order to solve the system we can use de Gauss Elimination, LU factorization, (see $[6,9]$ ) or Uzwa Method (see [6]).

Note that each square matrix of linear system is of $(m-1)$ th order, since every matrix defined by (32) is of order $(m-1)$. So the linear system is of order $2(m-1) \times 2(m-1)$, with the coefficient block matrix and the right-hand side known from the previous iteration.

\section{Uncoupled system}

Since $\left\{d^{n}, g^{n}\right\}$ must be solved simultaneously at each time step, the preceding numerical scheme is computationally coupled. From the numerical standpoint the coupled system is larger and hence harder to solve than an uncoupled system involving only $d^{n+1}$ or only $g^{n}$ at each time step $t_{n}$. In order to get uncoupled system, (see [1] and [12]), we replace the central difference by the backward extrapolation for the first derivative,

$$
d^{\prime}\left(t_{n}\right)=\frac{1}{2 \Delta t}\left(3 d^{n}-4 d^{n-1}+d^{n-2}\right)
$$


then by substituting it in the system (25) $)_{1,2}$ together with (29) and (30), we obtain, after some simple calculation,

$$
\begin{aligned}
& \widehat{A}^{n} d^{n+1}=\widehat{B}^{n} d^{n}-\widehat{C}^{n} d^{n-1}-\widehat{D}^{n} g^{n+1}-\widehat{E}^{n} g^{n}-\widehat{F}^{n} g^{n-1} \\
& \widetilde{A}^{n} g^{n+1}=-\widetilde{B}^{n} d^{n}+\widetilde{C}^{n} d^{n-1}-\widetilde{D}^{n} d^{n-2}-\widetilde{E}^{n} g^{n}+\widetilde{F}^{n} g^{n-1}
\end{aligned}
$$

where

$$
\begin{aligned}
\widehat{A}^{n} & =A+\delta \Delta t^{2} B_{1}^{n}+\frac{\Delta t}{2} D^{n}, & \widehat{B}^{n} & =2 A-\Delta t^{2}\left((1-2 \delta) B_{1}^{n}+B_{2}^{n}+E^{n}\right), \\
\widehat{C}^{n} & =A+\delta \Delta t^{2} B_{1}^{n}-\frac{\Delta t}{2} D^{n}, & \widehat{D}^{n} & =\delta a_{2}^{n} \Delta t^{2} C, \\
\widehat{E}^{n} & =(1-2 \delta) a_{2}^{n} \Delta t^{2} C, & \widehat{F}^{n} & =\delta a_{2}^{n} \Delta t^{2} C, \\
\widetilde{A}^{n} & =\frac{1}{2} A+\delta b_{1}^{n} \Delta t F, & \widetilde{B}^{n} & =\frac{1}{2}\left(3 b_{2}^{n}+4 b_{4}^{n} \Delta t\right) C+\Delta t R^{n}, \\
\widetilde{C}^{n} & =2 b_{2}^{n} C, & \widetilde{D}^{n} & =\frac{1}{4} \widetilde{C}^{n}, \\
\widetilde{E}^{n} & =\Delta t\left((1-2 \delta) b_{1}^{n} F+G^{n}\right), & \widetilde{F}^{n} & =\frac{1}{2} A-\delta b_{1}^{n} \Delta t F .
\end{aligned}
$$

We start the iteration by taking $n=0$ in $(36)_{2}$ and then taking $n=0$ in (36) $)_{1}$, to obtain

$$
\begin{aligned}
& \widetilde{A}^{0} g^{1}=-\widetilde{B}^{0} d^{0}+\widetilde{C}^{0} d^{-1}-\widetilde{D}^{0} d^{-2}-\widetilde{E}^{0} g^{0}+\widetilde{F}^{0} g^{-1} \\
& \widehat{A}^{0} d^{1}=\widehat{B}^{0} d^{0}-\widehat{C}^{0} d^{-1}-\widehat{D}^{0} g^{1}-\widehat{E}^{0} g^{0}-\widehat{F}^{0} g^{-1}
\end{aligned}
$$

The terms on the right-hand side of $(38)_{1}$, now involve the values $\left\{d^{0}, d^{-1}\right.$, $\left.d^{-2}, g^{0}, g^{-1}\right\}$ which are known by (33) and the term $d^{-2}$ calculated by taking $n=0$ in (35),

$$
d^{-2}=-3 d^{0}+4 d^{-1}+2 \Delta t d^{\prime}(0)
$$

Therefore, the value of $g^{1}$ is calculated in $(38)_{1}$ and the value of $d^{1}$ can be determined from $(38)_{2}$. Now we can go to iteration steps $n=1,2 \cdots, N$ similarly, by first solving $(36)_{2}$ then $(36)_{1}$ alternatively in each step. In this manner the numerical system is uncoupled in this computational scheme and we obtain the values $\left\{g^{n}, d^{n}\right\}$ for $n=1,2, \cdots N$. These values together with the starting values, constitute the finite element approximate solutions to the initial boundary value problem of Problem (II). 


\section{Numerical simulation}

A numerical example will be given to illustrate some features of the present model, using the method developed for the uncoupled system that is more efficient. In the example, we need the constants $\eta_{1}, \eta_{2}$ and $k$, which give rise to the coupling of the parabolic and hyperbolic equation in the thermoelastic system (I). The constants are given by the following formulas

$$
\eta_{1}=\frac{\bar{\alpha}(3 \lambda+2 \mu) \sqrt{\theta_{0}}}{\sqrt{(\lambda+2 \mu) \rho c l^{2}}}, \quad \eta_{2}=l \eta_{1}, \quad k=\frac{\bar{k}}{c l \sqrt{\rho(\lambda+2 \mu)}} .
$$

where $c$ is the specific heat; $\bar{\alpha}$ is the coefficient of thermal expansion; $\bar{k}$ is the thermal conductivity; $l=2 K(0)$ is the length of the string; $\rho$ is the density of the string; $\theta_{0}$ is the initial temperature; $\lambda$ and $\mu$ are the coefficients of Lamé,

$$
\mu=\frac{E v}{(1+v)(1-2 v)}, \quad \lambda=\frac{E}{2(1+v)},
$$

where $v$ is the coefficient of Poisson and E is the Young's modulus.

For the numerical example, these values will be calculated from the physical properties of aluminum. In this case, we have $\mu=26.24 \times 10^{9}$ and $\lambda=$ $58.41 \times 10^{9}$. Using the thermal and mechanical properties of aluminum, we obtain the approximate values $\eta_{1}=0.164, \eta_{2}=0.161$ and $k=0.177$.

Let us consider in (29) the weight $\delta=0.5$ and let $\Omega=(-K(t), K(t))$ be divided into $m$ subintervals, i.e, $h=2 / m$ and $\Delta t=T / N$, for different values of $N$ and $T$ for the discrete time. To calculate the coefficients defined in (24) in each step, the function $K(t)$ that defines the time dependence of the boundary for the non-cylindrical domain $Q_{t}$ in (20) must be given. In this example it is given by $K(t)=1-1 / \exp ^{(t+1)}$. Note that in this case, $Q_{t}$ tends to $Q$ rapidly as $t$ increases. This particular function is taken in order to satisfy the hypothesis $\mathbf{H 2}$, i.e, $K^{\prime}(t) \approx 1$. From the physical point of view, we require that the speed of the end points be less than the "characteristic" speed of the system.

Note that when only wave equations for small vibrations of elastic string or beam equation, both with moving boundaries, the monotonicity of those functions is not required (see [3], [8], [13]).

We consider the initial temperature, the initial position and velocity given by

$$
\phi_{0}(y)=0.033\left(1-y^{2}\right), \quad v_{0}(y)=0.057\left(y^{2}-1\right) \quad \text { and } \quad v_{1}(y)=0 .
$$


In all the figures the space variable is the axis- $x$, by the change of variable $y=(x-\alpha(t)) / \gamma(t))$.

For Fig. 1-Fig. 4 we have used $\Delta t=0.03$ and $h=0.02$, with $N=m=100$ and $T=3$. Fig. 1 and Fig. 2, respectively, shows the temperature $\theta(x, t)$ and the displacement $u(x, t)$ in the midpoint $x=0$.

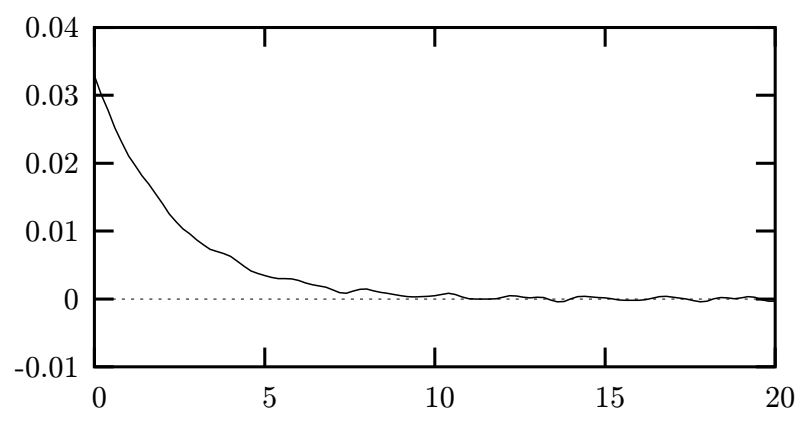

Figure 1 - Temperature at midpoint $\theta(0, t)$.

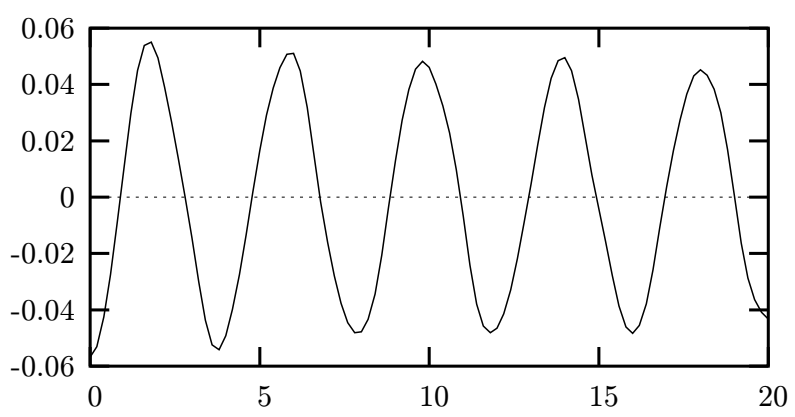

Figure 2 - Displacement at midpoint $u(0, t)$.

Fig. 3 and Fig. 4, show the approximate solutions $\theta\left(x, t^{*}\right)$ and $u\left(x, t^{*}\right)$, in the interval $[0, T]=[0,3]$ for different values of $t^{*}, t^{*}=0,0.25,0.75,1.5,2.25,3.0$.

Note that the interval of the boundary has varied from $[-0.63,0.63]$ to [-0.98, 0.98].

To obtain Fig. 5-Fig. 8, we have used $\Delta t=0.1$ and $h=0.04$. In Fig. 5 and Fig. 6 the evolution of the displacement function $u(x, t)$ is plotted, showing the profile of the displacement, where time varies from 0 to 5 and 0 to 10 , at 0.1 interval respectively. 


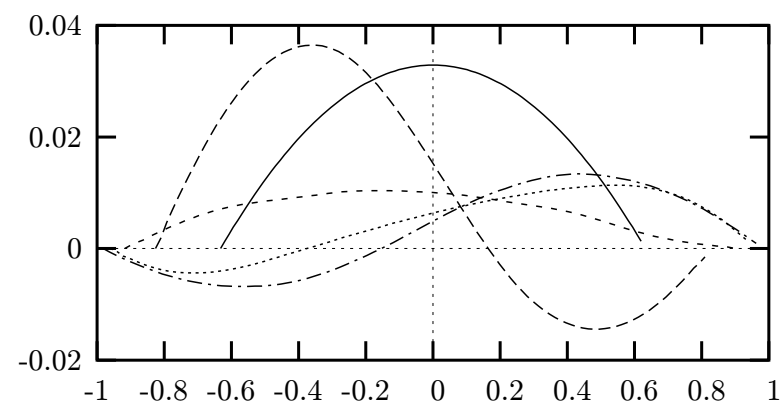

Figure $3-\theta\left(x, t^{*}\right)$ at $t^{*}=0,0.25,0.75,1.5,2.25,3.0$.

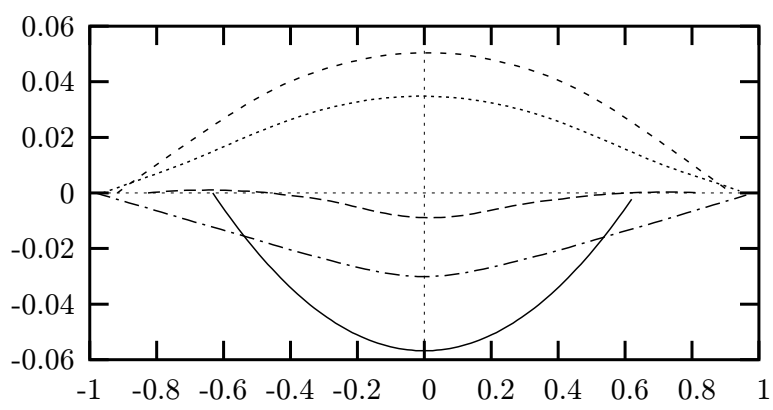

Figure $4-u\left(x, t^{*}\right)$ at $t^{*}=0,0.25,0.75,1.5,2.25,3.0$.

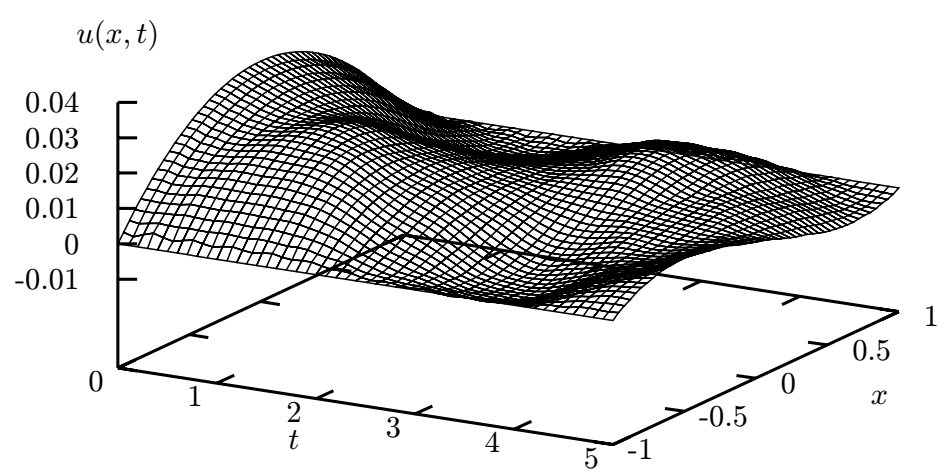

Figure 5 - Displacement with 50 time steps.

In Fig. 7 and Fig. 8 the evolution of the temperature function $\theta(x, t)$ are plotted, showing the profile of the temperature, where time varies from 0 to 5 and 0 to 10 , at 0.1 interval respectively. 


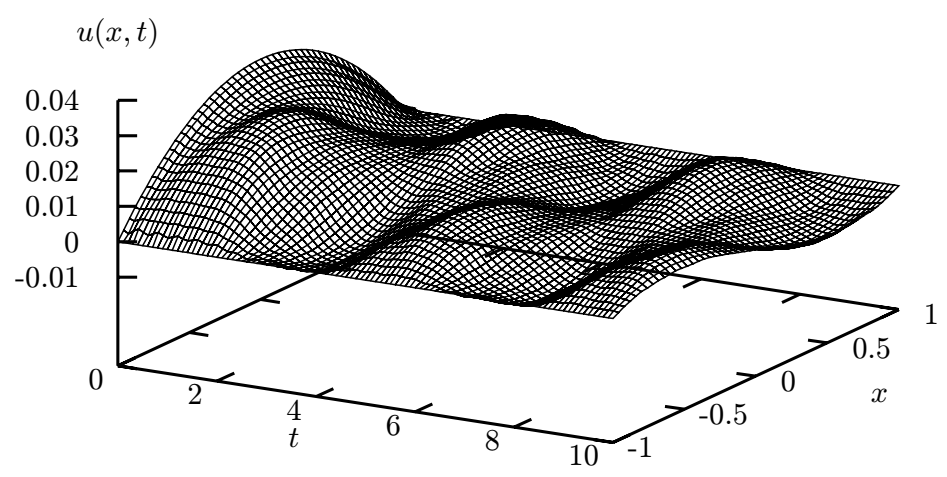

Figure 6-Displacement with 100 time steps.

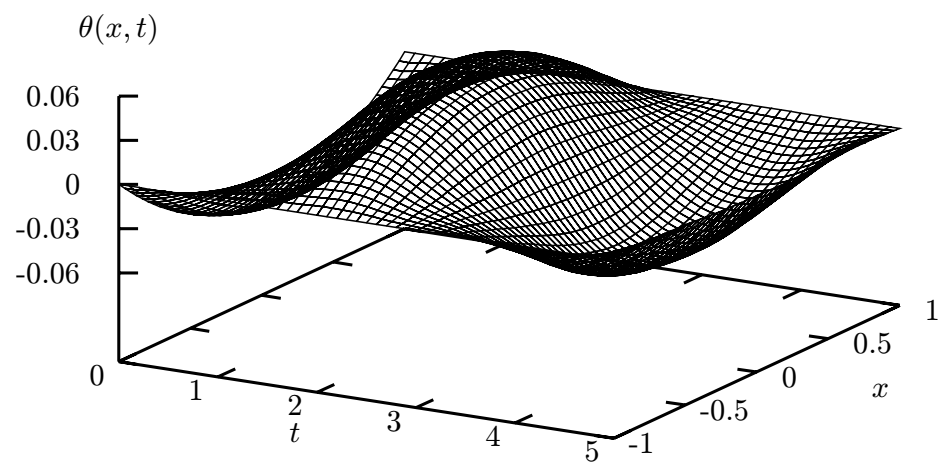

Figure 7 - Temperature with 50 time steps.

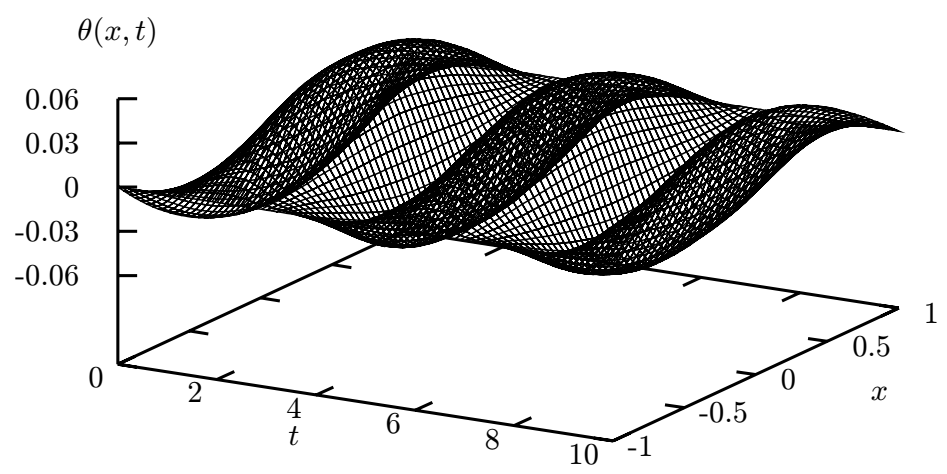

Figure 8 - Temperature with 100 time steps. 


\section{REFERENCES}

[1] S.I. Chou and C.C. Wang, Estimates of error in finite element approximate solutions to problems in linear thermoelsticity, Part II. Computationally uncoupled numerical schemes, Arch. Rational Mech. Anal., 77 (1981), 263-299.

[2] J. Clank, The Mathematics of Diffusion, 2rd ed., Oxford University Press (1975).

[3] H.R. Clark, M.A. Rincon and R.D. Rodrigues, Beam Equation with Weak-Internal Damping in Domain with Moving Boundary, Applied Numerical Mathematics, Vol. 47, Fasc. 2, (2003), 139-157.

[4] C.M. Dafermos, On the existence and the asymptotic stability of solution to the equations of linear thermoelasticity, Arch. Rational Mech. Anal., 29 (1968), 241-271.

[5] G. Dassios and M. Grillakis, Dissipation rates and partition of energy in thermoelasticity, Arch. Rational Mech. Anal., 87-1 (1984), 49-91.

[6] G.H. Golub and C.F Van Loan, Matrix Computations, 3 rd ed., Johns Hopkins U. Press, Bltimore, (1996).

[7] T.J.R. Hugles, The finite Element Method Linear Static and Dynamic Finite Element Analysis, Prentice Hall (1987).

[8] Liu, I-Shih and M.A. Rincon, Effect of Moving Boundaries on the Vibrating Elastic String, Applied Numerical Mathematics, Vol. 47, Fasc. 2, (2003), 159-172.

[9] Lloyd N. Trefethen and David Bau III, Numerical Linear Algebra, 1 rd ed., SIAM, Philadelphia, (1997)

[10] L.A. Medeiros, J. Límaco and S.B. Menezes, Vibrations of Elastic Strings: Mathematical Aspects, Part One, Journal of Computational Analysis and Applications, vol. 4 (2002), 91-127.

[11] L.A. Medeiros, J. Límaco and S.B. Menezes, Vibrations of Elastic Strings: Mathematical Aspects, Part two, Journal of Computational Analysis and applications, vol. 4 (2002), 211263.

[12] C.A. de Moura, A linear uncoupling numerical scheme for the nonlinear coupled thermoelastodynamics equations, Numerical Methods, V. Pereyra and A. Reinoza (eds.). Lecture Notes in Mathematics n. 1005, Springer-Verlag, 1983.

[13] M.A. Rincon, Liu; I-Shih, Existence and Uniqueness of Solutions of Elastic String with Moving Ends, Mathematical Methods in the Applied Sciences, Vol. 27 (2004), 1641-1655. 